\title{
Configural and featural processing during face perception: A new stimulus set
}

\author{
Goedele Van Belle, Michael De Smet, Peter De Graef, \\ LuC VAN GOOL, ANd KarL Verfaillie \\ University of Leuven, Leuven, Belgium
}

\begin{abstract}
A new stimulus set of 60 male-face stimuli in seven in-depth orientations was developed. The set can be used in research on configural versus featural mechanisms of face processing. Configural, or holistic, changes are produced by changing the global form of the face, whereas featural, or part-based, changes are attained by altering the local form of internal facial features. For each face in the set, there is one other face that differs only by its global form and one other face that differs only by its internal features. In all faces, extrafacial cues have been eliminated or standardized. The stimulus set also contains a color-coded division of each face in areas of interest, which is useful for eye movement research on face scanning strategies. We report a matching experiment with upright and inverted face pairs that demonstrates that the face stimulus set is indeed useful for research on configural and featural face perception. The stimulus set may be downloaded from the Psychonomic Society's archive (brm.psychonomic-journals.org/content/supplemental) or from our Web site (http://ppw.kuleuven .be/labexpsy/newSite/resources).
\end{abstract}

Human observers are experts in recognizing many different faces, even with different expressions, varying hair styles, and different attributes, such as jewelry, makeup, and glasses. This expertise is remarkable, since faces are such complex stimuli, differing only subtly in local features (e.g., the shape of the nose or mouth, eye color, etc.) and in configural, or holistic, characteristics (i.e., the spatial relations and global form of the face, which determine the perception of the face as a whole).

In the scientific community, there is general agreement that face perception, more than the perception of most other objects, relies mainly on configural/holistic, rather than on local/part-based, processing (Farah, Wilson, Drain, \& Tanaka, 1998; Maurer, Le Grand, \& Mondloch, 2002). Apart from recognizing the importance of configural processing in normal face perception, numerous studies have suggested that problems with face recognition in some syndromes (e.g., prosopagnosia, autism) might also be due to a failure of configural processing (Barton, Press, Keenan, \& O'Connor, 2002; De Gelder, Frissen, Barton, \& Hadjikhani, 2003; Joubert et al., 2003; Langdell, 1978; Levine \& Calvanio, 1989; Miyashita, 1988; Sergent \& Signoret, 1992; Sergent \& Villemure, 1989; Tantam, Monaghan, Nicholson, \& Stirling, 1989). However, despite the suggested central role of configural processing in both normal and disturbed face perception, there is an extensive discussion in the literature about the meanings and appropriate use of the terms configural face processing and holistic face processing (Rossion, 2008), as well as about the exact nature of the global and/or local cues that are involved in face-specific configural/holistic processing (Yovel \& Kanwisher, 2008). Here, we have no intention of participating in this debate; rather, we present a set of stimuli suitable for examining the effects of global versus part-based alterations of facial stimuli. We hope this set will then serve to further elucidate theoretical questions on configural versus featural processing of faces.

Experimental research about configural and featural face perception requires a set of standardized face stimuli in which global and part-based information can be manipulated independently. Moreover, it would be preferable to be able to register both types of changes at similar levels in the visual processing hierarchy. Barton et al. (2002), for example, developed a procedure for investigating the role of configural processing in face recognition by manipulating the distance between the eyes or the distance between the mouth and the nose. Featural processing is then manipulated by changing eye color. It could be questioned, however, whether eye-color changes and feature spacing changes are equivalent manipulations of facial information, given that they may be processed at different levels of the visual hierarchy (Yovel \& Kanwisher, 2004).

Taking these considerations into account, we present a stimulus set of natural-looking faces, in which recognition is possible only when it is based on the form of the facial features and the global facial form. All extrafacial cues have been eliminated or standardized. Furthermore, the face stimuli can be combined in pairs of faces, differing from each other in part-based features, global form, or both. 


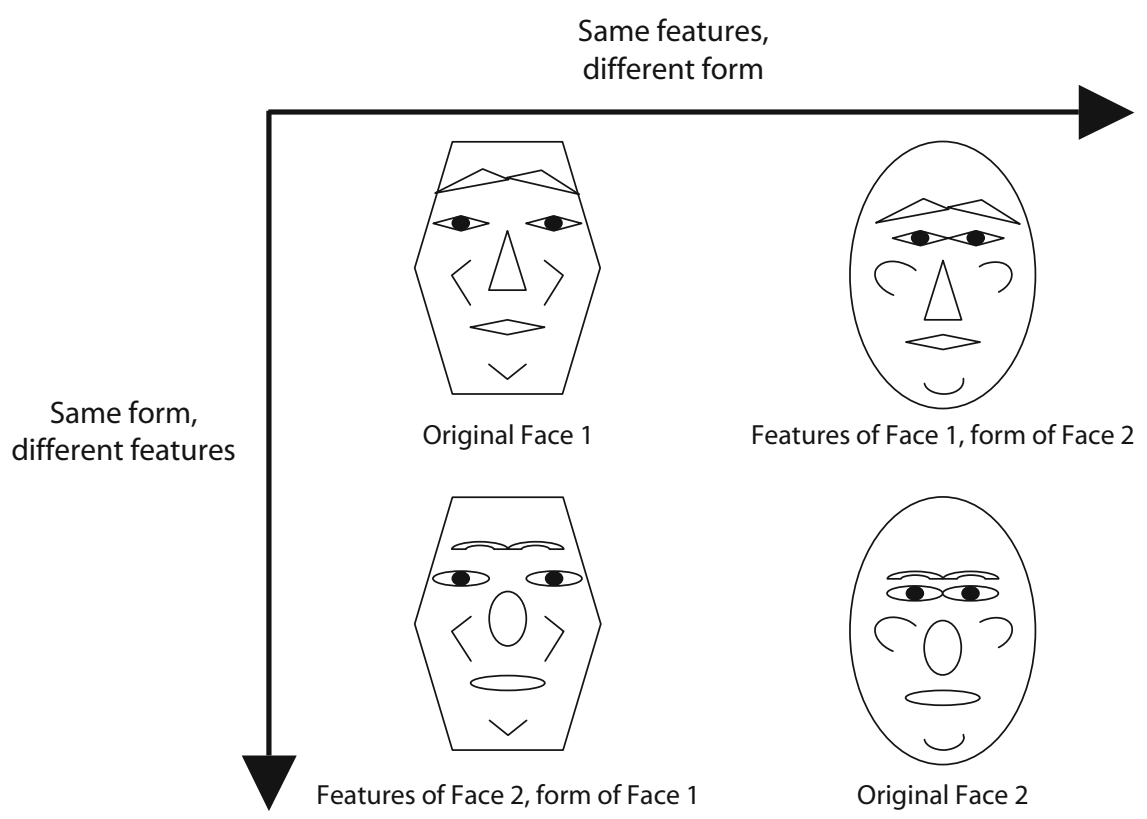

Figure 1. Schematic overview of the creation of the stimuli.

\section{The Stimulus Set}

A series of 30 Caucasian male faces was generated using a 3-D morphable model trained on the USF HumanID 3-D database (Blanz \& Vetter, 1999). All faces shared the same skin structure and color (an average of the skin structures and colors from the faces in the USF HumanID 3-D database), thereby preventing the use of skin structure or color as a basis for identification. No extrafacial cues, such as hair, color differences, makeup, clothing, or jewelry, were present. In this way, the only remaining differences among the faces were in global form-determined by the form of the skull, muscles, and fat structure - and in the form of their internal facial features, such as the eyes, nose, and mouth. The faces appeared on a uniform, gray background.

As is schematically shown in Figure 1, the original 30 faces were combined into 15 pairs. From each pair of faces, two new variants were created by separating and crossing the global form and the internal features of the two faces. The global form of each face was obtained by iteratively smoothing the 3-D shape until the fine-scale structure was removed. The internal features were subsequently determined by subtracting the global form from the original 3 -D shape of the face. In this manner, the first variant for each pair involved a global transformation produced by applying the skull, muscles, and fat structure from one face in the pair to the other. The internal facial features were fully preserved. It is important to note that this type of global transformation was not limited to merely altering the spacing of internal facial features, as has been the case in many preceding studies of featural versus configural face perception (e.g., Yovel \& Duchaine, 2006). In the second variant for each pair, a local part-based transformation was produced by merging the internal features from one face in the pair with the unchanged global form of the other face. An example of the result is shown in Figure 2. In total, this procedure resulted in a set of 60 faces. For all faces, seven different profile views are available.

Furthermore, for each face in the stimulus set, subdivision into color-coded areas is available. An example is presented in Figure 3. The color-coded areas can serve as areas of interest in eye movement research on face scanning strategies (see, e.g., Barton, Radcliffe, Cherkasova, \& Edelman, 2007).

The file names of the faces in the stimulus set contain all the information about their features, global form, and orientation, as explained in full detail in the included README file. The stimulus set can be downloaded from http:// ppw.kuleuven.be/labexppsy/newSite/resources and from the Psychonomic Society's archive at brm.psychonomic -journals.org/content/supplemental.

\section{The Present Experiment}

The main goal of the present experiment was to determine whether our manipulation of the stimuli would be useful for research on configural and featural face perception. More specifically, we sought to determine whether observers would be able to detect both part-based and global form changes in the stimulus set. If they could not, this would mean that the differences in form or in features between the stimuli are not large enough to allow observers to discriminate between identical and different faces, and that the stimuli, therefore, would not be useful for face discrimination tasks.

Furthermore, we investigated whether the stimuli could be used to distinguish between configural and featural information processing. To achieve this, we used a phenomenon known as the face inversion effect (Yin, 1969). Turning images upside down disproportionately disrupts face recognition, as compared with the recognition of 


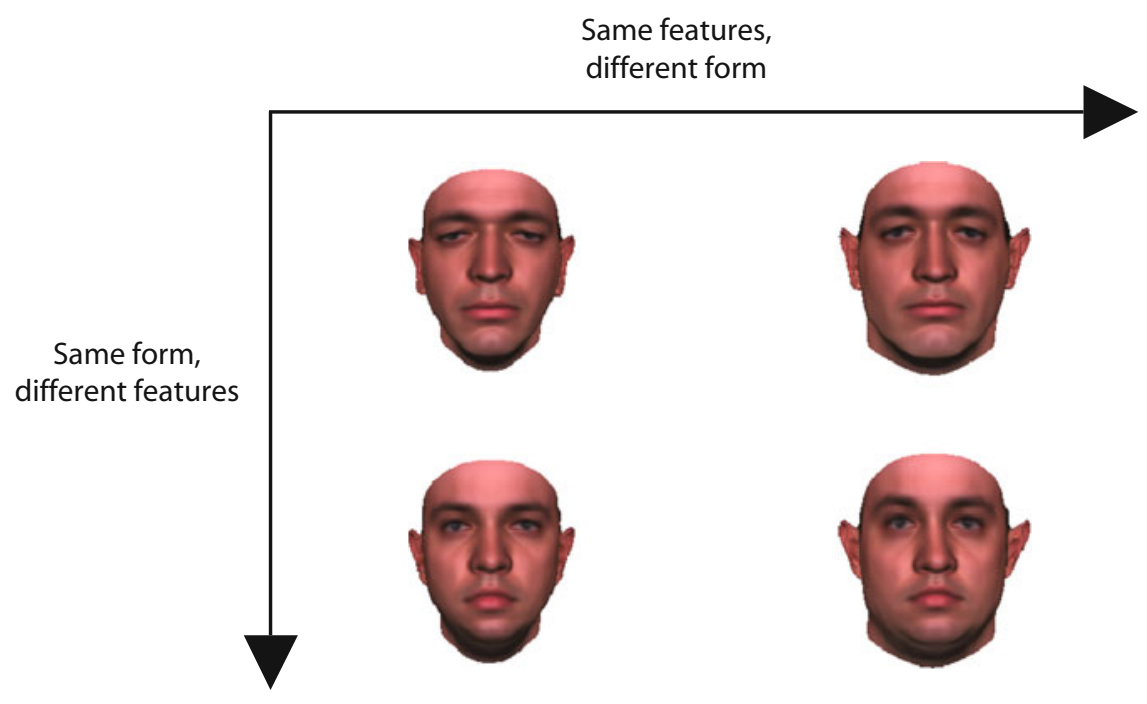

Figure 2. A set of real stimuli. Skin color and structure are averaged over the skin colors and structures of the faces in the USF HumanID 3-D database.

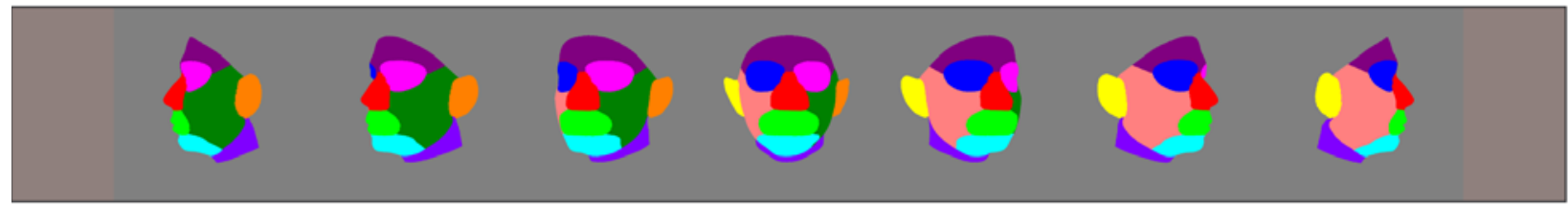

Figure 3. The subdivision of facial parts.

most other objects (Rossion \& Gauthier, 2002; Valentine, 1988). It has been suggested that the face inversion effect might result from the inability of the face expert system to process configural information in faces when they diverge from their normal orientation (e.g., Leder \& Bruce, 1998; Rhodes, Brake, \& Atkinson, 1993; Schwaninger \& Mast, 2005; Searcy \& Bartlett, 1996; Sergent, 1984). In our stimulus set, configuration differences are a direct consequence of the applied changes in the global facial form, whereas the part-based feature changes have far more subtle configurational consequences (if any); we therefore hypothesized a larger face inversion effect in detecting global form differences than in detecting local feature form differences. Importantly, this does not rule out inversion effects on the discrimination of facial feature differences, as has been suggested in a number of recent studies (e.g., Malcolm, Leung, \& Barton, 2004; Riesenhuber, Jarudi, Gilad, \& Sinha, 2004; Yovel \& Duchaine, 2006; Yovel \& Kanwisher, 2004; but see Rossion, 2008 , for a methodological critique of these findings). We merely want to determine whether plausible gradations in the strength of configural facial differences can be produced with the present set of stimuli.

\section{METHOD}

\section{Participants}

One hundred thirteen first-year psychology students of the University of Leuven participated in the experiment for course credit.
They all had normal or corrected-to-normal visual acuity and were naive as to the purpose of the study.

\section{Stimuli and Procedure}

The participants were tested in groups of about 30 students, seated in a PC lab at approximately $50 \mathrm{~cm}$ from the computer screen. The stimuli were displayed using a Dell Optiplex GX280 with a standard 17-in. monitor, controlled by E-Prime software (Psychological Software Tools, Pittsburgh, PA). The monitor had a spatial resolution of $1,280 \times 1,024$ pixels and a refresh rate of $80 \mathrm{~Hz}$. As presented in Figure 4, each trial consisted of two faces, side by side. The participants' task was to indicate whether they thought the two faces belonged to the same person or not by pressing one of two buttons on the keyboard. The participants were instructed to look very carefully, because some faces belonged to family members and could be very similar without being equal. Stimuli were presented until response.

In order to avoid pixelwise comparison between the faces, one face was oriented to the viewer; the other face was oriented $30^{\circ}$ away from the frontal view. One of the faces was slightly smaller than the other (with a scaling factor of 1.1) in order to prevent participants from basing decisions on mere picture height differences. To avoid any effects of afterimages, and to clearly separate consecutive trials, a 400-msec random-dots mask was presented between trials.

Two blocks of 40 trials each were presented to the participants. In one of the blocks, the faces were presented upright, whereas in the other one, they were inverted. Of the 40 trials in each block, there were 10 from each of the following conditions: (1) equal, (2) same features but different form, (3) different features but same form, and (4) different features and different form. The order of the trials within the blocks was randomized. The faces were selected randomly, so that none of the participants got the same face-pair combination twice. The order of the blocks was counterbalanced. 
A

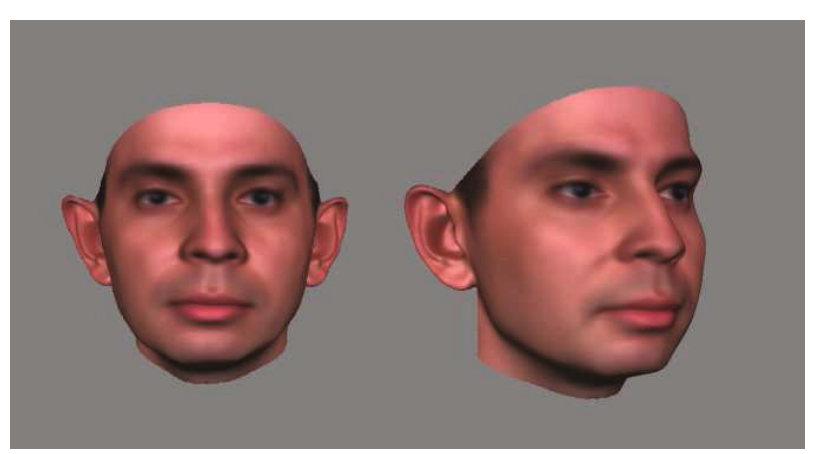

B

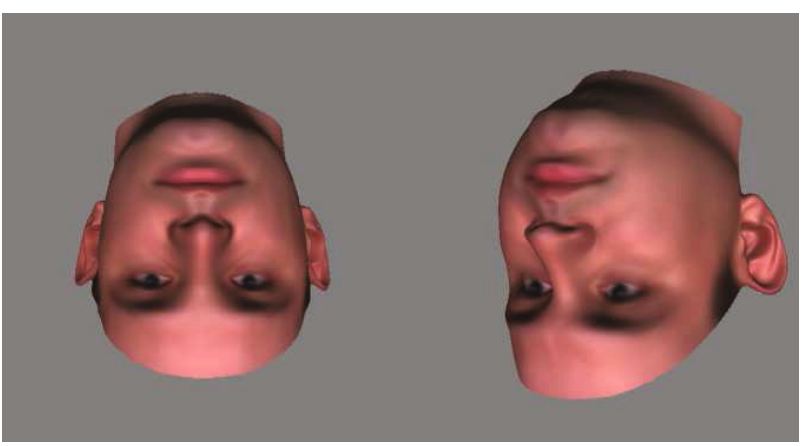

Figure 4. Face pairs in (A) the upright condition (equal face pair) and (B) the inverted condition (completely different face pair).

\section{RESULTS AND DISCUSSION}

For each participant and each experimental condition, we calculated $d^{\prime}$, a bias-free sensitivity index (Macmillan $\&$ Creelman, 1991). A $d^{\prime}$ of 0 indicates chance level. The average $d^{\prime}$ in each condition can be found in Figure 5. Hits were defined as trials in which the participants correctly noticed that two faces were different; false alarms were trials in which the participants incorrectly judged equal faces to be different. Each trial took about $3 \mathrm{sec}$, on average.

Our first goal was to verify whether feature differences and form differences in the stimuli were detectable. Therefore, we investigated whether participants' performance was above chance level in all conditions. We found that all $d^{\prime}$ 's were significantly larger than $0(p<.01)$. This means that, even in the condition in which the differences were hardest to detect, the differences were not too small to be detectable.

The second goal was to examine whether the manipulation of the global form and of the form of the features would reflect the expected gradations in the prominence of configural versus featural processing. A repeated measures ANOVA revealed a main effect of orientation of the faces $\left[F(1,112)=67.60, M S_{\mathrm{e}}=79.99, p<.0001\right]$. On average, performance for the upright faces $\left(d^{\prime}=1.19\right)$

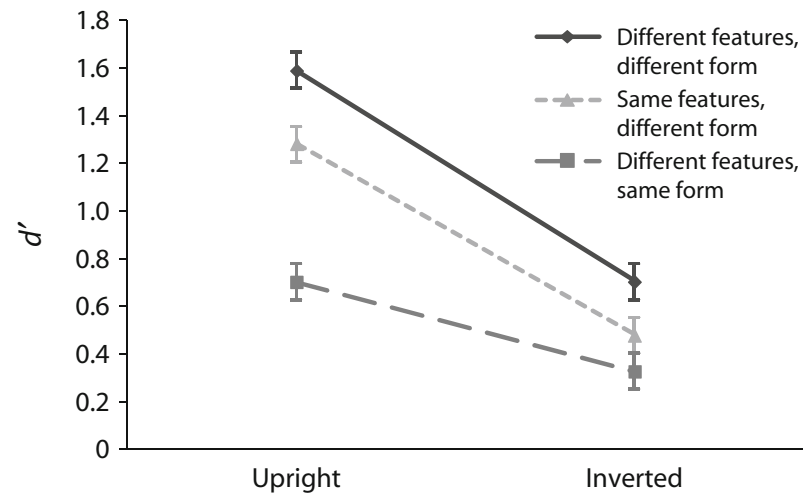

Figure 5. The average $d^{\prime}$ for all conditions. The error bars indicate the standard errors. was better than that for the inverted faces $\left(d^{\prime}=0.50\right)$. The interaction of orientation and feature and form differences on task performance also proved significant $[F(2,224)=$ $\left.25.71, M S_{\mathrm{e}}=4.20, p<.0001\right]$. The inversion effect turned out to be much larger in both conditions with global form differences than in the condition with only local feature differences. Note that the difference in performance for the upright face conditions does not invalidate this conclusion. First, even for the most difficult condition, performance was significantly above chance, ruling out a floor effect. Second, so far no reliable correlation between upright performance level and size of the inversion effect has been established (Rossion, 2008). Taking these together, we therefore believe that the present data provide convincing evidence that the global form manipulation in the proposed stimulus set indeed causes more configural changes than does the feature manipulation.

\section{Conclusions}

The face stimulus set was shown to be useful for research on configural and featural face perception. All types of differences were large enough to be detected. Furthermore, global form manipulations resulted in a more pronounced face inversion effect than did feature manipulations, suggesting that the stimuli are suitable for examining within- and across-populations differences in the degree of configural versus featural face processing.

\section{AUTHOR NOTE}

This study was supported by the Flemish Government through FWO Grant 7.0005.05, by IWT-Flanders through the AMASS++ Project (SBO-IWT nb. 060051), by the IBBT-ISBO Project PISA, and by the European Community through GazeCom Project IST-C-033816. We are grateful to Sudeep Sarkar of the University of South Florida for allowing us to use the USF HumanID 3-D database in this research. Correspondence concerning this article should be addressed to G. Van Belle, PSI 00.70, Laboratory of Experimental Psychology, University of Leuven, Tiensestraat 102, B-3000 Leuven, Belgium (e-mail: goedele .vanbelle@psy.kuleuven.be).

\section{REFERENCES}

Barton, J. J. S., Press, D. Z., Keenan, J. P., \& O’Connor, M. (2002). Lesions of the fusiform face area impair perception of facial configuration in prosopagnosia. Neurology, 58, 71-78. 
Barton, J. J. S., Radcliffe, N., Cherkasova, M. V., \& Edelman, J. A. (2007). Scan patterns during the processing of facial identity in prosopagnosia. Experimental Brain Research, 181, 199-211.

Blanz, V., \& Vetter, T. A. (1999). A morphable model for the synthesis of 3D faces. Proceedings of SIGGRAPH 99, 187-194.

De Gelder, B., Frissen, I., Barton, J. [J. S.], \& Hadjikhani, N. (2003) A modulatory role for facial expressions in prosopagnosia. Proceedings of the National Academy of Sciences, 100, 13105-13110.

Farah, M. J., Wilson, K. D., Drain, M., \& TanaKa, J. N. (1998). What is "special" about face perception? Psychological Review, 105, 482-498.

Joubert, S., Felician, O., Barbeau, E., Sontheimer, A., Barton, J. J. [S.], Ceccaldi, M., \& Poncet, M. (2003). Impaired configurational processing in a case of progressive prosopagnosia associated with predominant right temporal lobe atrophy. Brain, 126, 2537-2550.

LANGDELL, T. (1978). Recognition of faces: An approach to the study of autism. Journal of Child Psychology \& Psychiatry, 19, 255-268.

LEDER, H., \& BRUCE, V. (1998). Local and relational aspects of face distinctiveness. Quarterly Journal of Experimental Psychology, 51A, 449-473.

Levine, D. N., \& Calvanio, R. (1989). Prosopagnosia: A defect in visual configural processing. Brain \& Cognition, 10, 149-170.

Macmillan, N. A., \& Creelman, C. D. (1991). Detection theory: A user's guide. New York: Cambridge University Press.

Malcolm, G. L., LeunG, C., \& Barton, J. J. S. (2004). Regional variation in the inversion effect for faces: Differential effects for feature shape, feature configuration, and external contour. Perception, $\mathbf{3 3}$ 1221-1231.

Maurer, D., Le Grand, R., \& Mondloch, C. J. (2002). The many faces of configural processing. Trends in Cognitive Sciences, 6, 255-260.

Miyashita, T. (1988). Discrimination of facial components in autistic children. Japanese Journal of Psychology, 59, 206-212.

Rhodes, G., Brake, S. \& AtKinson, A. P. (1993). What's lost in inverted faces? Cognition, 47, 25-57.

Riesenhuber, M., JARUdi, I., Gilad, S., \& Sinha, P. (2004). Face processing in humans is compatible with a simple shape-based model of vision. Proceedings of the Royal Society B, 271, 448-450.

Rossion, B. (2008). Picture-plane inversion leads to qualitative changes of face perception. Acta Psychologica, 128, 274-289.

Rossion, B., \& GAUTHIER, I. (2002). How does the brain process upright and inverted faces? Behavioral \& Cognitive Neuroscience Reviews, 1, 63-75.
Schwaninger, A., \& MAST, F. W. (2005). The face-inversion effect can be explained by the capacity limitations of an orientation normalization mechanism. Japanese Psychological Research, 47, 216-222.

SeARCY, J. H., \& BARTlett, J. C. (1996). Inversion and processing of component and spatial-relational information in faces. Journal of Experimental Psychology: Human Perception \& Performance, 22, 904-915.

SERGENT, J. (1984). An investigation into component and configural processes underlying face perception. British Journal of Psychology, 75, 221-242

Sergent, J., \& Signoret, J.-L. (1992). Varieties of functional deficits in prosopagnosia. Cerebral Cortex, 2, 375-388.

Sergent, J., \& Villemure, J.-G. (1989). Prosopagnosia in a right hemispherectomized patient. Brain, 112, 975-995.

Tantam, D., Monaghan, L., Nicholson, H., \& Stirling, J. (1989). Autistic children's ability to interpret faces: A research note. Journal of Child Psychology \& Psychiatry, 30, 623-630.

Valentine, T. (1988). Upside-down faces: A review of the effect of inversion upon face recognition. British Journal of Psychology, 79, 471-491.

YIN, R. K. (1969). Looking at upside-down faces. Journal of Experimental Psychology, 81, 141-145.

Yovel, G., \& Duchaine, B. (2006). Specialized face perception mechanisms extract both part and spacing information: Evidence from developmental prosopagnosia. Journal of Cognitive Neuroscience, 18, 580-593.

Yovel, G., \& Kanwisher, N. (2004). Face perception: Domain specific, not process specific. Neuron, 44, 889-898.

Yovel, G., \& Kanwisher, N. (2008). The representations of spacing and part-based information are associated for upright faces but dissociated for objects: Evidence from individual differences. Psychonomic Bulletin \& Review, 15, 933-939.

\section{SUPPLEMENTAL MATERIALS}

The 839 pictures of faces in this study may be downloaded as supplemental materials for this article from brm.psychonomic-journals.org/ content/supplemental.

(Manuscript received July 17, 2008; revision accepted for publication October 1, 2008.) 